\title{
Effects of Reynolds Number and Flapping Kinematics on Hovering Aerodynamics
}

\author{
Jian Tang,,$*$ Dragos Viieru, $₫$ and Wei Shyy \\ University of Michigan, Ann Arbor, Michigan 48109
}

DOI: $\underline{10.2514 / 1.32191}$

\begin{abstract}
Motivated by our interest in micro and biological air vehicles, Navier-Stokes simulations for fluid flow around a hovering elliptic airfoil have been conducted to investigate the effects of Reynolds number, reduced frequency, and flapping kinematics on the flow structure and aerodynamics. The Reynolds number investigated ranges from $\mathbf{7 5}$ to 1700 , and the reduced frequency from 0.36 to 2.0. Two flapping modes are studied, namely, the "water-treading" hovering mode, and the normal hovering mode. Although the delayed-stall mechanism is found to be responsible for generating the maximum lift peaks in both hovering modes, the wake-capturing mechanism is identified only in the normal hovering mode. In addition to the strong role played by the kinematics, the Reynolds number's role has also been clearly identified. In the low Reynolds number regime, $\mathcal{O}(\mathbf{1 0 0})$, the viscosity dissipates the vortex structures quickly and leads to essentially symmetric flow structure and aerodynamics force between the forward stroke and backward strokes. At higher Reynolds numbers (300 and larger), the history effect is influential, resulting in distinctly asymmetric phenomena between the forward and backward strokes.
\end{abstract}

\section{Nomenclature}

$C_{d}$

$C_{f}$

$C_{l}$

$c$

$f$

$h$

$h_{a}$

$U_{\infty}$ $u, v, w$

$x, y, z$

$x_{m}, x_{s}$

$\alpha$

$\alpha_{a}$

$\alpha_{0}$

$\mu$

$\xi, \eta, \zeta$

$\rho$

$\boldsymbol{\tau}_{i, j}$

$\boldsymbol{\tau}_{w}$

$v$

$\varphi$

$=\mathrm{d}$

$=$ chord length

= flapping frequency

= flapping amplitude

$=$ Jacobian

$=$ reduced frequency

$=$ static pressure

$=$ Reynolds number

$=$ period of one flapping cycle

$=$ reference velocity

$=$ freestream reference velocity

$=$ Cartesian coordinates

$=$ angle of attack

$=$ pitching angle amplitude

$=$ initial angle of attack

$=$ coefficient of viscosity

$=$ curvilinear coordinates

$=$ density

$=$ viscous stress

$=$ wall shear stress

$=$ kinematic viscosity $=\quad \operatorname{drag} /\left(0.5 \rho c U_{\infty}^{2}\right)$

$=$ friction coefficient $=\operatorname{drag} /\left(0.5 \rho c U_{\infty}^{2}\right)$

$=$ lift coefficient $=$ lift $/\left(0.5 \rho c U_{\infty}^{2}\right)$

$=$ instantaneous position of flapping airfoil

$=$ nondimensionalized time $=t c / U_{\text {ref }}$

$=$ Cartesian velocity components

$=$ Cartesian coordinates of master/slave nodes

$=$ phase difference between flapping and pitching motion

Presented as Paper 129 at the 45th AIAA Aerospace Sciences Meeting and Exhibit, Reno, Nevada, 8-11 January 2007; received 16 May 2007; accepted for publication 27 October 2007. Copyright (C) 2007 by the American Institute of Aeronautics and Astronautics, Inc. All rights reserved. Published by the American Institute of Aeronautics and Astronautics, Inc., with permission. Copies of this paper may be made for personal or internal use, on condition that the copier pay the $\$ 10.00$ per-copy fee to the Copyright Clearance Center, Inc., 222 Rosewood Drive, Danvers, MA 01923; include the code 0001-1452/ $08 \$ 10.00$ in correspondence with the CCC.

*Post Doctoral Research Fellow, Department of Aerospace Engineering; jiantang@umich.edu. Member AIAA.

${ }^{\dagger}$ Post Doctoral Research Fellow, Department of Aerospace Engineering; dviieru@umich.edu. Member AIAA.

†Clarence L. "Kelly" Johnson Collegiate Professor and Chair, Department of Aerospace Engineering; weishyy@umich.edu. Fellow AIAA. $\omega=$ oscillating angular speed $=2 \pi f$

Superscript

$=$ nondimensional quantities

\section{Introduction}

W ITH our desire to understand the capabilities of natural flyers such as birds, bats, and insects, and, lately, with increasing interest in developing the micro air vehicle technologies, substantial research efforts have been made on flapping flight. For example, Shyy et al. [1] reviewed the overall background of the micro and natural air vehicles including the scaling laws and the associated research from the computational and modeling viewpoints. Lehmann [2] , Norberg [3] , Jones and Platzer [4] , Viieru et al. [5] , and Wang [6] offered reviews on flapping wing aerodynamics from different angles. Mueller [7] compiled a number of articles contributed by different authors regarding the analysis and design of fixed and flapping wing flying vehicles.

Natural flyers operate in a wide range of Reynolds numbers and flapping frequencies, while employing different kinematics for the wing movement. For example, a pigeon flies at a Reynolds number, based on the forward flight velocity, of $10^{5}$ while beating its wings at nearly $6 \mathrm{~Hz}$ [8]. Bumblebees operate at a Reynolds number around $4 \times 10^{3}$ and flapping frequencies of $156 \mathrm{~Hz}$ [8], while dragonflies, flying at similar Reynolds numbers, flap their wings only at around $29 \mathrm{~Hz}$ [8]. At the lower end of the spectrum, one can find the fruit flies and chalcid wasps flying at Reynolds numbers of 200 and, respectively, 20 while the flapping frequency reaches $\mathcal{O}(100) \mathrm{Hz}$ [8] . A correlation between a flyer's mass and the reduced frequency [5] based on data from Azuma [8], Pennycuick [9], and Tennekes [1이] shows that, overall, the reduced frequency increases as the size and mass decreases indicating that unsteadiness plays an important role in small flyer's movement. Ellington [11] pointed out, in a comprehensive analysis, that the aerodynamic forces in flapping flight predicted by classical, steady-state aerodynamic theories have been found insufficient to explain the insect/bird flight characteristics. Therefore, unsteady effects have an important role in aerodynamic force generation. Four unsteady physical mechanisms have been identified so far in the literature to help explain how insects and birds generate enhanced lift, namely, Weis-Fogh's clap-and-fling mechanism [2,12], delayed stall associated with large scale vortices [13] and fast pitch-up [14], and wake capturing [15]. These lift generation mechanisms have been identified experimentally and 
numerically. For example, Liu and Kawachi [16] conducted unsteady Navier-Stokes simulations of the flow around a hawk moth's wing. Their results showed the leading-edge vortex and the spanwise flow observed experimentally by Van den Berg and Ellington [17] and Ellington et al. [13]. Sun and Tang [18] and Ramamurti and Sandberg [19] confirmed the force peaks generated during fast pitch-up of the wing at the end of the stroke, and the wake-capturing mechanisms identified experimentally by Dickinson et al. [15].

As the flyers' sizes and their flight Reynolds number vary, the relative importance among viscous, convective, and pressure terms changes accordingly, which exerts substantial impact on the aerodynamics and fluid physics as suggested in [5]. Despite the importance of 3-D effects, comparison of experiments and computations in 2-D has provided important insight and might be sufficient to explain some enhanced lift coefficients measured in insects. Wang et al. [20] compared computational, experimental, and quasi-steady forces in a generic hovering wing, undergoing sinusoidal motion along a horizontal stroke plane, to examine the unsteady aerodynamics. The computed forces were compared with the threedimensional experimental results of Dickinson et al. [15]. In a recent paper, Kurtulus et al. [21] investigated the relation between the temporal and spatial changes of the wake structure and the resulting instantaneous aerodynamic forces over a plunging and pitching NACA 0012 airfoil and different parameters such as angle of attack, kinematics, and Reynolds number. They found that for angles of attack greater than $30 \mathrm{deg}$, positive lift is obtained throughout the motion.

The main objective of this study is to better understand the physical mechanisms associated with the Reynolds number, reduced frequency, and flapping kinematics so that we can gain more insight into the way they interact and impact the aerodynamics. In this paper, Navier-Stokes simulations for fluid flow around a hovering elliptic airfoil have been conducted to investigate the effects of Reynolds number, reduced frequency, and flapping kinematics on the flow structure and aerodynamics. The Reynolds number investigated ranges from 75 to 1700 , and the reduced frequency from 0.25 to 2.0. To help facilitate the investigation of the kinematics effect, two flapping modes are studied, namely, the "water-treading" hovering mode and the "normal" hovering mode. The water-treading mode, as defined by Freymuth [22], is used mainly in aquatic locomotion, while the normal hovering mode as referred to by Weis-Fogh [12] is employed by most insects during hovering. Both modes are characterized by symmetric back-and-forth strokes in a horizontal stroke plane and they are described in detail in the paper. In the following, we first briefly describe the numerical algorithm used to solve the flow equations, as well as the moving grid strategy employed. The numerical framework is validated against established analytical and computational results. Then, the fluid physics and the aerodynamic implications are probed based on the cases selected.

\section{Numerical Algorithm}

The three-dimensional Navier-Stokes equations for incompressible flow in Cartesian coordinates can be written, using indicial notation, as follows:

$$
\begin{gathered}
\frac{\partial \rho}{\partial t}+\frac{\partial}{\partial x_{j}}\left(\rho u_{j}\right)=0 \\
\frac{\partial}{\partial t}\left(\rho u_{i}\right)+\frac{\partial}{\partial x_{j}}\left(\rho u_{j} u_{i}\right)=-\frac{\partial P}{\partial x_{i}}+\frac{\partial \tau_{i j}}{\partial x_{j}}
\end{gathered}
$$

where $x_{i}$ is the position vector, $t$ is time, $\rho$ is density, $u_{i}$ is the velocity vector, $p$ is pressure, and $\tau_{i j}$ is the viscous stress tensor. The constitutive relation between stress and strain rate for a Newtonian fluid is used to link the components of the stress tensor to velocity gradients:

$$
\tau_{i j}=\mu\left(\frac{\partial u_{i}}{\partial x_{j}}+\frac{\partial u_{j}}{\partial x_{i}}\right)-\frac{2}{3} \mu \frac{\partial u_{l}}{\partial x_{l}} \cdot \delta_{i j}
$$

where $\mu$ is the molecular viscosity.
For arbitrary shaped geometries, the Navier-Stokes equations are transformed into generalized curvilinear coordinates $(\xi, \eta, \zeta)$, where, $\xi=\xi(x, y, z), \eta=\eta(x, y, z)$, and $\zeta=\zeta(x, y, z)$. The transformation of the physical domain $(x, y, z)$ to the computational domain $(\xi, \eta, \zeta)$ is achieved by the following relations [23]:

$$
\left[\begin{array}{lll}
\xi_{x} & \xi_{y} & \xi_{z} \\
\eta_{x} & \eta_{y} & \eta_{z} \\
\zeta_{x} & \zeta_{y} & \zeta_{z}
\end{array}\right]=\frac{1}{J}\left[\begin{array}{lll}
f_{11} & f_{12} & f_{13} \\
f_{21} & f_{22} & f_{23} \\
f_{31} & f_{32} & f_{33}
\end{array}\right]
$$

where $f_{i j}$ are the metric terms and $J$ is the determinant of the Jacobian transformation matrix given by

$$
J=\operatorname{det}\left(\frac{\partial(x, y, z)}{\partial(\xi, \eta, \zeta)}\right)=\operatorname{det}\left[\begin{array}{ccc}
x_{\xi} & x_{\eta} & x_{\zeta} \\
y_{\xi} & y_{\eta} & y_{\zeta} \\
z_{\xi} & z_{\eta} & z_{\zeta}
\end{array}\right]
$$

To solve for the Navier-Stokes equations in curvilinear coordinates, the finite volume formulation is adopted. In this approach, both Cartesian velocity, as primary variables, and contravariant velocity components are employed. The contravariant velocities are used to evaluate the flux at the cell faces and to enforce the mass continuity in the pressure-correction equation. The expressions for the metrics, the determinant of the Jacobian transformation matrix, and the fluxes at the cell faces as well as for the three-dimensional Navier-Stokes equations in the generalized bodyfitted curvilinear coordinate system $(\xi, \eta, \zeta)$ are given in [24,25]. When the governing equations are considered under a moving grid framework, the grid velocities should be included in the flux computations as described in [1].

The pressure-correction equation developed by Patankar and Spalding [26] and enhanced by Van Doormaal and Raithby [27] was extended to the curvilinear coordinates, with a hybrid employment of the Cartesian and contravariant velocity components (see Shyy et al. [28]). The implementation of the current method employs a control volume approach and uses a nonstaggered arrangement for the velocity components and the scalar variables (i.e., pressure). The Cartesian velocity components are computed from the respective momentum equations. The cell fluxes and pressure fields are corrected using a pressure-correction equation, which is derived by manipulating the continuity and momentum equations. The iterative correction procedure leads to a divergence-free velocity field within a desired convergence tolerance, therefore enforcing the pressurevelocity coupling.

To solve the governing equations in a body-fitted curvilinear coordinate, a transformation matrix is used to facilitate the mapping of a physical flow region $(x, y, z)$ onto a computational domain $(\xi, \eta, \zeta)$. The Jacobian transformation matrix is defined as in Eq. (5). The determinant of the Jacobian transformation matrix represents the volume element in the transformed coordinate. In moving grid problems, the computational grid is changing with time and consequently the determinant of the Jacobian matrix $J$ needs to be updated. Special procedures are required to compute the effective value of $J$ at each time step; otherwise, errors arise due to an inconsistent numerical implementation that would lead to the generation of artificial mass sources. As suggested by Thomas and Lombard [29], in the process of updating the Jacobian $J$, the following geometric conservation law needs to be satisfied:

$$
\frac{\mathrm{d}}{\mathrm{d} t} \int_{V} J \mathrm{~d} \xi \mathrm{d} \eta \mathrm{d} \zeta=\int_{V}\left(\nabla \cdot W_{s}\right) \mathrm{d} \xi \mathrm{d} \eta \mathrm{d} \zeta
$$

where $V$ is the volume bounded by the closed surface $S$, and $W_{s}$ is the local velocity of the moving boundary surface $S$. Thomas and Lombard [29] proposed an expression to evaluate $J$ from the continuity equation for a constant density, uniform velocity field under a time-dependent coordinate transformation while maintaining the geometric conservation law:

$$
\frac{\partial J}{\partial t}=\frac{\partial}{\partial \xi}\left(J \frac{\partial \xi}{\partial t}\right)+\frac{\partial}{\partial \eta}\left(J \frac{\partial \eta}{\partial t}\right)+\frac{\partial}{\partial \zeta}\left(J \frac{\partial \zeta}{\partial t}\right)
$$


Integrating Eq. (7) using a first-order, implicit time integration scheme over the same control volume used for mass conservation leads to a finite volume discrete form of the above equation that is used to update the Jacobian in a manner that respects the basic requirement of the geometric conservation in the discrete form of the conservation law when the grid is time dependent. Implications of the geometric conservation law on the moving boundary problems were discussed by Shyy et al. [28].

For moving boundary problems where a solid boundary (i.e., airfoil) moves inside a computational domain based on known kinematics (i.e., rigid flapping airfoil) or as a response of the structure to the flow around it (i.e., membrane wing), the grid needs to be adjusted dynamically during computation. To facilitate this, a moving grid technique needs to be employed. The actual process of generating a grid is a complicated task by itself so an automatic and fast algorithm to upgrade the grid frequently is essential. It is desirable to have an automatic remeshing algorithm to ensure that the dynamically moving grid retains the quality of the initial grid and avoids problems such as crossover of the grid lines, crossed cell faces, or negative volumes at block interfaces in the case of multiblock grids.

Several approaches have been suggested to treat grid redistributions for moving grid computations. Schuster et al. [30] used an algebraic shearing method in their study. The displacement of the moving surface is redistributed along the grid line which connects the moving surface to the outer boundary. This simple method gives good results for modest displacement and single block grid. For multiblock grid arrangements extensive user intervention is required. A robust method that can handle large deformations is the spring analogy method that was first introduced by Batina [31] for unstructured grids and later extended to structured grids by Robinson et al. [32]. In this method all edges of a cell as well as the diagonals are replaced by linear springs, each with the stiffness inversely proportional to a power $p$ of the length of the connecting edges. Using the power $p$, one can control the stiffness of the spring and consequently control the amount of movement and avoid excessive mesh distortions. The iterative process necessary to find the displacement of all the internal points increases the computational time for this method, especially for large grids. The direct transfinite interpolation method was introduced by Eriksson [33] and can generate grids for complex geometry. The method defines an interpolation function given known values on constant planes and function derivatives in out of surface direction on the boundaries. The method is fast and efficient for structured grids but the quality of the initial grid may not always be preserved especially far from the boundaries. Hartwich and Agrawal [34] propose the master-slave concept to expedite the grid regeneration process and preserve the grid continuity at the multiblock grid interfaces.

In this study a moving grid technique proposed by Lian et al. [35] is used to remesh the multiblock structured grid for fluid-structure interaction problems. For multiblock structured grids, for simplicity, computational fluid dynamics solvers often require point-matched grid block interfaces. This method is based on the master-slave concept and maintains a point-matched grid block interface while maintaining grid quality and preventing potential grid crossover.

When an object changes its shape, the master points, which are located at the moving body surface, move first, and then affect the distribution of the off-body points. One difficulty for a multiblock grid resides in the way in which the vertices of each block are moved if a point-to-point match between two abutting blocks without overlap is required. For identical interfaces between two abutting blocks, the edge and interior points can be obtained by a 3-stage interpolation once the corner vertices are determined. However, when the abutting blocks do not have an identical interface, the interpolation can cause discontinuity at the interface. To avoid creating undesirable grid discontinuities, the off-body vertices of a grid block are linked to a surface point and thus they move in a similar way. Therefore, for each off-body vertex (slave point), the nearest body surface point is defined as its master point. The distance between the slave point and its master is given by

$$
|\boldsymbol{r}|=\sqrt{\left(x_{s}-x_{m}\right)^{2}+\left(y_{s}-y_{m}\right)^{2}+\left(z_{s}-z_{m}\right)^{2}}
$$

where the subscript $s$ represents a slave point, and $m$ a master point. Once a slave point has its master point identified, the slave point moves according to the influence from its master.

The master-slave algorithm is highly automated, maintaining overall grid quality near and away from the body and more important allows instantaneous large displacements. The propagation distance of the moving wall perturbation is controlled by the spring stiffness coefficient. However, in the current formulation large rotational deformations cannot be handled properly because no information is provided about cell skewness as the perturbation is propagated along grid lines. To solve this problem, a modification is made to add the rotational angle to the position information in the original algorithm.

\section{Results and Analysis}

\section{Code Validation}

To validate the present formulation, three cases are studied. First, the steady flow over a flat plate at zero angle of attack at different Reynolds numbers representative to insect flapping flight is computed. The evaluated friction coefficient is compared with the analytical results. Second, the flow over an oscillating flat plate in a quiescent medium is simulated to assess the viscous force computation for moving walls. Third, the two-dimensional simulated flow around a flapping wing is contrasted with existing experimental and other computational results.

To simulate the steady flow over a thin plate of chord $c=1.0$, the Navier-Stokes equations are solved on a grid with 100 points along the plate and 60 points in the vertical direction. The distance from the wall to the first cell center is $5.0 \times 10^{-4} c$, which guarantees a sufficient number of points in the boundary layer for high Reynolds numbers. The numerical simulations are performed for Reynolds numbers from 10 to $10^{4}$, based on chord length and freestream velocity. A second-order upwind scheme was employed for the convection terms, while a second-order central difference scheme is adopted for the pressure and viscous terms. A no-slip boundary condition is imposed on the plate surface.

In Fig. 1 the numerical and analytical velocity distribution in the boundary layer for a flat plate at different Reynolds numbers is plotted. As discussed by Schlichting [36], the velocity profile can be defined based on the following similarity form:

$$
u=U_{\text {ref }} f^{\prime}(n)
$$

where $U_{\text {ref }}$ is the freestream velocity, $f(\eta)$ is the Blasius solution, and $\eta=y \sqrt{U_{\text {ref }} /(v \cdot x)}$ is the dimensionless coordinate.

Figure 1 shows progressively favorable agreement between the numerical results and the analytical formulation, as the Reynolds number increases. This is expected because the boundary layer solution is based on the high Reynolds number assumption. The analysis of the flow over a flat plate using Blasius's equation is restricted to a semi-infinite plate because the parabolic nature of Prandtl's boundary layer equations cannot account for upstream changes in shear stress initiated by the trailing edge of a finite length plate. The discrepancy between the analytical and numerical velocity distribution is more visible at locations near the trailing edge of the plate as one can observe in Fig. 1 for all Reynolds numbers.

Figure 2 shows also that as the Reynolds number decreases, the friction coefficient given by Blasius departs from the numerical solution. To take into account second-order effects, generalizations of Prandtl's boundary layer equations were developed. For the case of the flat plate, Stewartson [37] and Messiter [38] found an expression that improves the prediction of the skin-friction coefficient for low Reynolds number. The analytical expressions for skin-friction coefficient, defined for two-dimensional flow as $C_{f}=\mathrm{drag} /\left(0.5 \rho c U_{\mathrm{ref}}^{2}\right)$, are presented in Table $\underline{1}$.

Figure 2 shows very good agreement between the improved analytical solution of Messiter [38] and the numerical results for a wide range of Reynolds numbers, validating the viscous force computation method employed in the solver. 


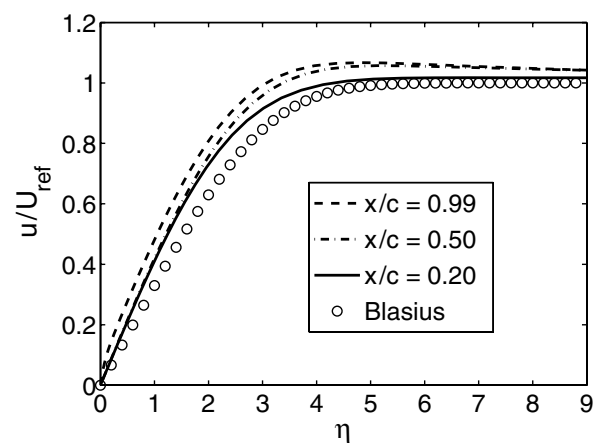

a)

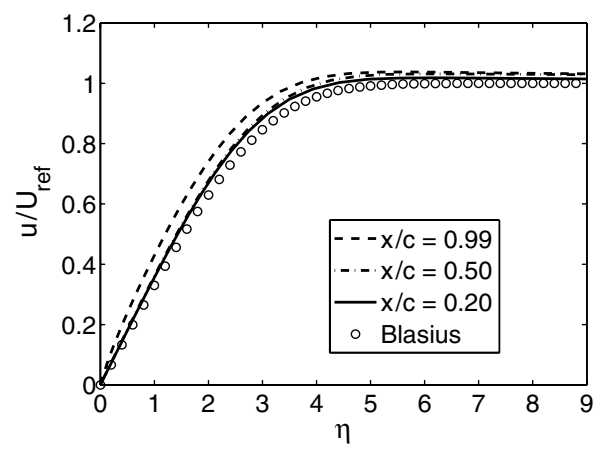

b)

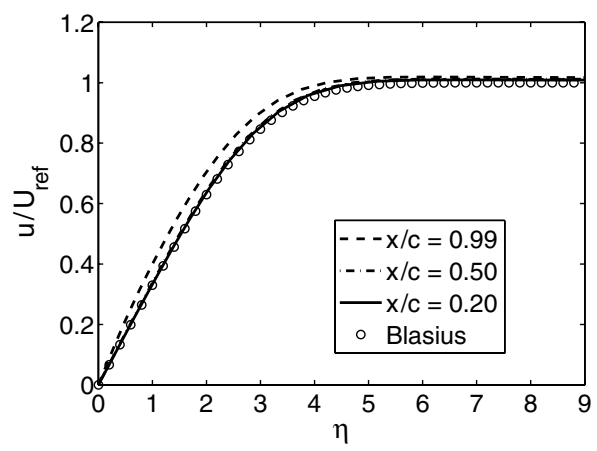

c)

Fig. 1 Numerical and analytical velocity distribution in the boundary layer along different locations on a flat plate and different Reynolds numbers. a) $R e=10^{2}$; b) $R e=10^{3}$; c) $R e=10^{4}$. Here is the $\eta=$ $y \sqrt{U_{\text {ref }} /(v \cdot x)}$ dimensionless vertical coordinate.

Next, the viscous force computation needs to be validated for the case of moving walls (wall velocity is nonzero). The wall velocity for an infinite plate oscillating along the $x$ axis is defined as

$$
u(y=0, t)=U_{\text {ref }} \cos (\omega t)
$$

where $U_{\text {ref }}$ is the maximum velocity, and $\omega=2 \pi f$, with $f$ being the oscillation frequency. The displacement of the plate is given by

$$
h(t)=h_{a} \sin (\omega t)
$$

where $h_{a}=U_{\text {ref }} / \omega$.

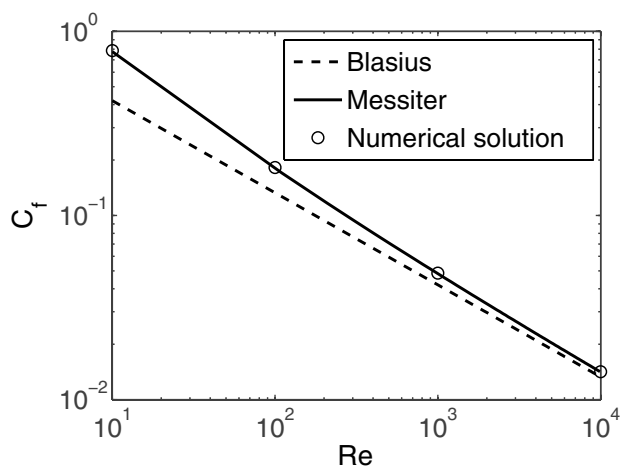

Fig. 2 Numerical and analytical friction coefficient $C_{f}$ for a flat plate versus Reynolds number. [39]:

The analytical velocity field for this motion is given by Stokes

$$
u / U_{\text {ref }}=\exp (-\eta) \cos (\omega t-\eta)
$$

where $\eta=y \sqrt{\omega / 2 v}$ is the nondimensional vertical coordinate.

Following the definition [Eq. (12)], the analytical wall shear stress for the oscillating plate is given by [으]

$$
\tau_{\text {wall }}=U_{\text {ref }} \sqrt{\rho \omega \mu} \cdot \sin (\omega t-\pi / 4)
$$

The unsteady flow over an oscillating plate of length 1 is solved on a grid with 50 points along the chord and 80 points in the vertical direction using the Navier-Stokes solver. The distance from the wall to the first cell center is $1.0 \times 10^{-3}$.

For the case studied, $\omega=2$, resulting in a frequency $f$ of $1 / \pi$ and a period $T=\pi$. To ensure a sufficient number of points for one oscillation cycle, a time step size of 0.01 is chosen. Based on the maximum velocity $U_{\text {ref }}$ and chord length, the Reynolds number is $10^{3}$.

In Fig. 3 the numerically predicted wall shear stress on the finite plate is compared with the analytical values for an infinite plate given by Eq. (13). The figure shows good agreement between numerical and analytical values. The small phase shift between numerical and analytical results can be explained by the effects of the leading and trailing edges of a finite plate. In Fig. 4 the scaled wall velocity is also plotted to contrast the lag between maximum shear and maximum velocity.

The theoretical velocity distribution above the plate, given by Eqs. (3) and (4), is plotted along with the computed velocity field in Fig. 4 for different time instants during one half-period. The skin friction and velocity profiles presented in Figs. 3 and 4 show good agreement between theoretical and computed results.

\section{Flapping Airfoil Solutions}

The main focus of this study is the investigation of aerodynamics of hovering flight. All cases are based on an elliptic airfoil of $15 \%$ thickness. Two hovering modes, namely, the water-treading mode and the normal hovering mode and the dimensionless parameters are described below. To insure the convergence of the solution, the time step size is chosen to result in nearly 900 time steps per flapping cycle. The computations show that 10 to 100 internal iterations per time step are needed to reach a global residual of $1.0 \mathrm{e}-04$. The present computations are done for multiple cycles. The results are

Table 1 Numerical and analytical friction coefficient values for a flat plate

\begin{tabular}{ccccc}
\hline \hline Case & Reynolds no. & $\begin{array}{c}C_{f}: \text { Blasius }(1904) \\
C_{f}=1.328 / \sqrt{R e_{l}}\end{array}$ & $\begin{array}{c}C_{f}: \text { Messiter }(1970)[38] \\
\left(C_{f}=1.328 / \sqrt{R e_{l}}+2.668 /\left(R e_{l}\right)^{7 / 8}\right)\end{array}$ & $\begin{array}{c}C_{f}: \text { present } \\
\text { numerical results }\end{array}$ \\
\hline 1 & $10^{4}$ & 0.0133 & 0.0141 & 0.0142 \\
2 & $10^{3}$ & 0.0420 & 0.0483 & 0.0486 \\
3 & $10^{2}$ & 0.133 & 0.180 & 0.182 \\
4 & 10 & 0.420 & 0.776 & 0.785 \\
\hline \hline
\end{tabular}




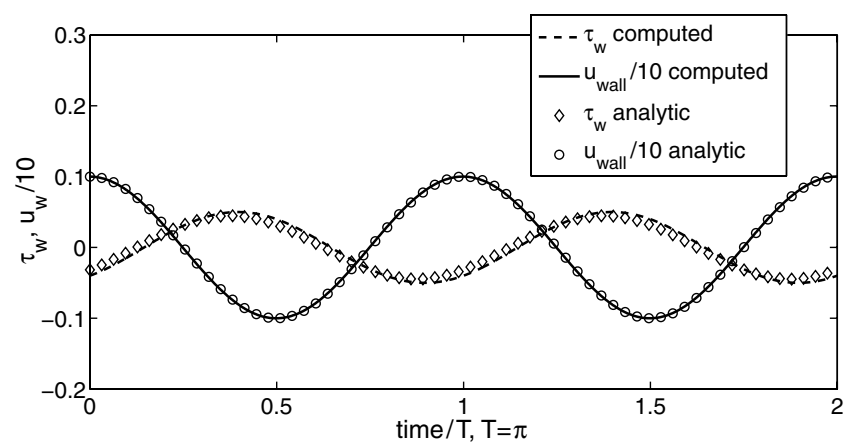

Fig. 3 Skin friction and wall velocity for an oscillating plate. The oscillating frequency is $f=\omega / 2 \pi=1 / \pi$, amplitude $h_{a}=0.5$, and $R e=10^{3}$. The wall velocity is scaled to an order of magnitude comparable to the wall shear stress. Continuous lines: present computational results; symbols: analytical solution for the infinite plate.

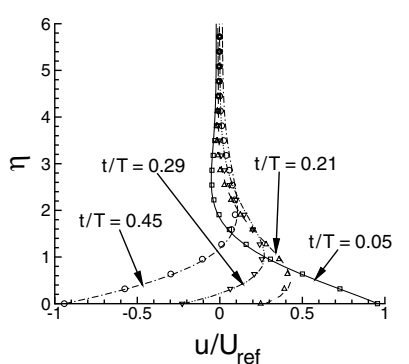

Fig. 4 Velocity distribution above an oscillating plate. The oscillating frequency is $f=\omega / 2 \pi=1 / \pi$, amplitude $h_{a}=0.5$, and $R e=10^{3}$. Continuous lines: present computational results; symbols: analytical solution for the infinite plate.

shown for a representative cycle, after the solution periodicity is achieved. In general, for all cases presented in this paper, a periodic behavior was observed after 4-6 cycles.

\section{Kinematics of the 2-D Hovering Modes}

Regarding the flapping kinematics, the airfoil's instantaneous location and incidence are uniquely defined by its translational and rotational coordinates, namely,

$$
\begin{gathered}
h(t)=h_{a} \sin (2 \pi f t) \\
\alpha(t)=\alpha_{0}+\alpha_{a} \sin (2 \pi f t+\varphi)
\end{gathered}
$$

where $h(t)$ is the instantaneous plunging amplitude, $h_{a}$ is plunging amplitude, normalized by the chord, $\alpha(t)$ is the instantaneous angle of attack, measured with respect to the horizontal line, $\alpha_{0}$ is the initial angle of attack, $\alpha_{a}$ is the pitching amplitude, and $\varphi$ is the phase difference between the plunging and pitching motion. For flow around a rigid, hovering airfoil with no freestream, there are two dimensionless parameters, namely, the reduced frequency and the Reynolds number. The reduced frequency $k$ is defined as

$$
k=2 \pi f c / 2 \quad U_{\text {ref }}=c / 2 h_{a}
$$
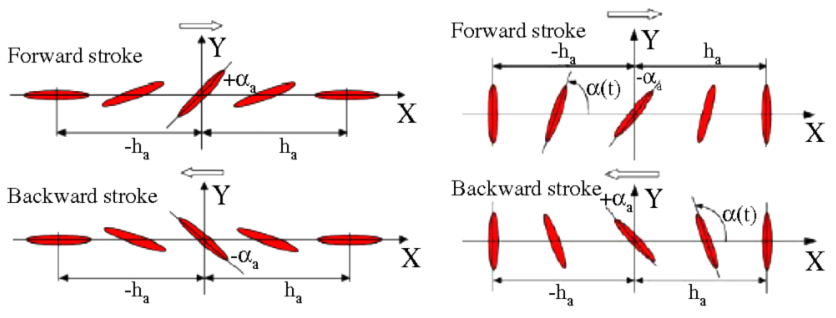

a)

b)

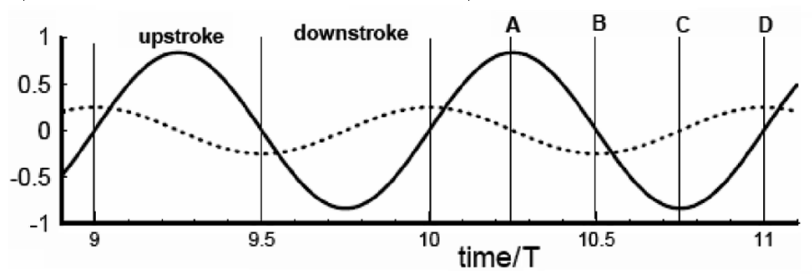

c)

Fig. 5 Basic characteristics of the hovering kinematics considered in this study. a) schematic of the water-treading mode; b) schematic of the "normal mode"; c) time histories of the airfoil stroke (solid line: $\boldsymbol{h}_{t}$ ) and pitching angle (dashed line: $\alpha_{t}$ ) employed for both modes in the present study.

where $c$ is the airfoil chord length, $f$ is the oscillation frequency, and $U_{\text {ref }}=2 \pi f h_{a}$ is the reference velocity (equal to the maximum plunging velocity). The Reynolds number is defined as

$$
\operatorname{Re}=U_{\mathrm{ref}} c / v
$$

It should be noted in Eq. (16) that the reduced frequency, by definition, varies with the inverse of the stroke amplitude. If we choose $c, U_{\text {ref }}$, and $1 / f$ as the length, velocity, and time scales, respectively, for nondimensionalization, then the corresponding momentum equation with constant density yields

$$
\frac{k}{\pi} \frac{\partial}{\partial \bar{t}}\left(\bar{u}_{i}\right)+\frac{\partial}{\partial \bar{x}_{j}}\left(\bar{u}_{j} \bar{u}_{i}\right)=\frac{\partial \bar{P}}{\partial \bar{x}_{j}}+\frac{1}{R e}\left(\frac{\partial^{2} \bar{u}_{i}}{\partial \bar{x}_{j}^{2}}+\frac{\partial^{2} \bar{u}_{j}}{\partial \bar{x}_{i}^{2}}\right)
$$

Two flapping modes have been investigated. The water-treading hovering mode is one of the cases that Liu and Kawachi [16] used to validate their finite volume algorithm. The hovering mode studied is based on the so-called water-treading mode as defined by Freymuth in his experiments [22]. Figure $\underline{5}$ depicts the overall characteristics of the water-treading motion. Furthermore, a normal hovering mode (Wang et al. [20]), depicted in Fig. 5, has also been studied.

Various cases involving the two flapping modes, different Reynolds numbers, and reduced frequencies have been computed. Table 2 summarizes these cases, which will be discussed in the following.

\section{Normal Hovering Mode at $\boldsymbol{R e}=\mathbf{1 0 0}$}

As reviewed by Wang [6], the normal mode, in which the wing moves in a level plane, is a mode popularly employed by insects and small birds in hovering. The unsteady, laminar, incompressible, Navier-Stokes equations are solved in an O-type domain around a $15 \%$ thickness elliptic airfoil. The spatial accuracy of the present algorithm is examined by employing three grid sizes. The coarse size

Table 2 Parameters considered in the present study

\begin{tabular}{lccccc}
\hline \hline Reynolds no. & 75 & 100 & 300 & 500 & 1700 \\
\hline Normal flapping & $h_{a} / c=0.25,1.4$ & $h_{a} / c=1.4$ & $h_{a} / c=0.25$ & $h_{a} / c=0.25$ & \\
& $(k=2,1 / 2.8)$ & $(k=1 / 2.8)$ & $(k=2)$ & $(k=2)$ & - \\
Water treading & - & $h_{a} / c=1.4$ & - & - & $h_{a} / c=1.4$ \\
& & $(k=1 / 2.8)$ & - & & $(k=1 / 2.8)$ \\
\hline \hline
\end{tabular}




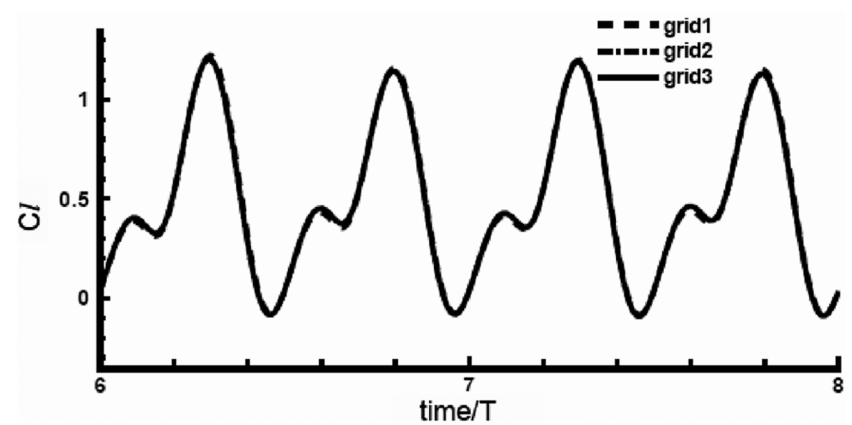

Fig. 6 Lift coefficient of the normal hovering mode for two periods and different grid sizes and $\delta t=0.01, h_{a} / c=1.4, \alpha_{a}=45 \mathrm{deg}, k=1 / 2.8$, and $R e=75$.

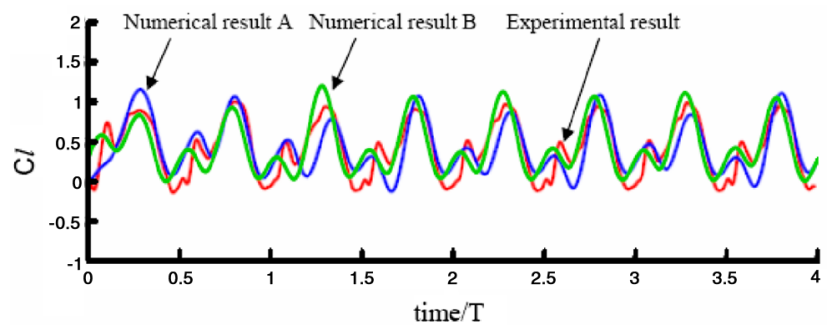

Fig. 7 Numerical and experimental results of the flapping motion of a fruit fly. Experimental result: experimental results of Dickinson and Birch (adopted from [20]). Numerical result A: numerical results of Wang (adopted from [20]). Numerical result B: present numeric simulation. $h_{a} / c=1.4, \alpha_{a}=45 \mathrm{deg}, k=1 / 2.8$, and $R e=75$. Here the lift force is normalized by the maximum quasi-steady force as defined by Wang et al. [20].

grid has $81 \times 81$ points (grid 1 ), while the intermediate size grid has $161 \times 161$ points (grid 2), and the fine grid size is $241 \times 241$ (grid 3). The distance from the solid wall to the first grid point is $0.001 c$. Consistent with the work of Wang et al. [20], a sinusoidal motion for both plunging and pitching motions is employed, and the airfoil rotation is symmetric, that is, the center of rotation is the center of the elliptic airfoil. The flapping motion and the rotational motion are described by Eqs. (14) and (15), and a schematic of the normal hovering mode is presented in Fig. $\underline{5}$.

In this case, the initial angle of attack is $\alpha_{0}=90 \mathrm{deg}$, the pitching amplitude $\alpha_{0}=45 \mathrm{deg}$, the nondimensional stroke amplitude $h_{a} / c=1.4$ (corresponding to a reduced frequency of $1 / 2.8$ ), and the phase lag is $\varphi=90 \mathrm{deg}$. According to Eq. (16), the reduced frequency is $1 / 2.8$ and the Reynolds number is 75 .

The flapping and pitching history is plotted in Fig. 5. The flapping and rotation of the up- and downstrokes in each cycle have the same absolute value and opposite signs, exhibiting a symmetric pattern. In Fig. 6, the lift coefficient history for two periods is plotted. At a Reynolds number of 75, a periodic pattern is noticed after four periods. Because there is little difference between the solutions on fine and intermediate grids, it is concluded that a grid independent solution was obtained.

Figure 7 compares our computational results with the results of Wang et al., and with the experimental results of Birch and Dickinson [20]. The current results show good agreement with the experimental measurements. Figure 7 shows that the lift patterns in forward and backward strokes of each flapping cycle are essentially unchanged, indicating that the effects of nonlinearity (convection) and history are modest.

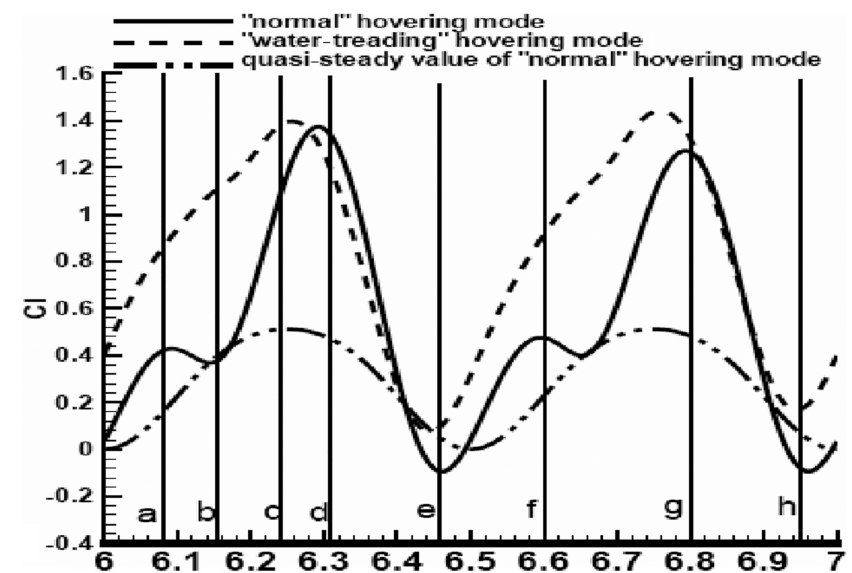

a)

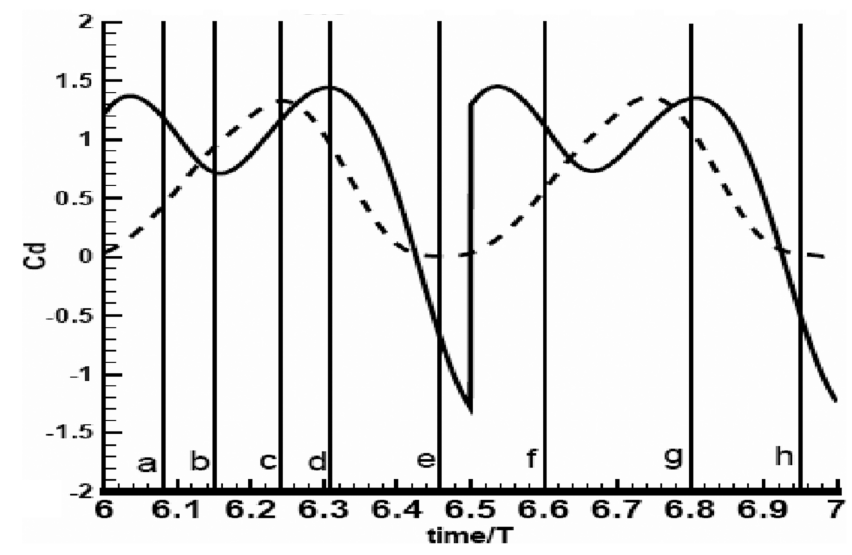

b)

Fig. 8 One cycle force history for two hovering modes and quasi-steady value of normal hovering mode. $h_{a} / c=1.4, \alpha_{a}=45 \mathrm{deg}, k=1 / 2.8$, and $R e=100$. a) Lift coefficient; b) drag coefficient. The selected normalized time instants are a) 6.08 , b) 6.17 , c) 6.25 , d) 6.31 , e) 6.45 , f) 6.60, g) 6.80, and h) 6.94 .

\section{Lift Generation Mechanisms in Two Hovering Modes}

The aerodynamic force generation by the same $15 \%$ thickness elliptic airfoil undergoing two different hovering modes is studied. First, the water-treading mode (Freymuth [22]) is considered, followed by the normal hovering mode. The water-treading mode is defined by Eqs. (14) and (15) and a schematic of this hovering mode is shown in Fig. 5 a. The motion of the normal hovering mode is governed by the same Eqs. (14) and (15) and is depicted in Fig. 5b. To compare the two hovering modes, consistent kinematics parameters are selected, as presented in Table 3 .

Figure 8 shows the lift and drag coefficients during one complete cycle for water-treading and normal hovering modes. To illustrate the unsteady effects, the quasi-steady value of normal hovering mode, as defined by Eqs. $(16,17)$ in $[20]$, is also plotted together.

In the case of water-treading hovering mode, for the first half of the forward stroke, the airfoil accelerates and pitches up. During this interval, the lift increases constantly (Fig. $\underline{8 a}$, times b, c), and the unsteady dynamics results in delayed flow separation even at instantaneously high angles of attack, as indicated by the vorticity contours plotted in Figs. 9a-9c.

The maximum lift is reached when the airfoil is close to the middle of the half-stroke, which is around the instant when the pitch angle

Table 3 Kinematic parameters for "water-treading" and "normal" hovering modes. The Reynolds number for both cases is 100

\begin{tabular}{lccccc}
\hline \hline Hovering mode & Initial angle of attack, $\alpha_{0}$ & Pitching amplitude, $\alpha_{a}$ & Stroke amplitude, $h_{a} / c$ & Reduced frequency, $k$ & Phase difference, $\varphi$ \\
\hline Water-treading hovering & $0 \mathrm{deg}$ & $45 \mathrm{deg}$ & 1.4 & $1 / 2.8$ & $-\pi / 2$ \\
Normal hovering & $90 \mathrm{deg}$ & $45 \mathrm{deg}$ & 1.4 & $1 / 2.8$ & $\pi / 2$ \\
\hline \hline
\end{tabular}




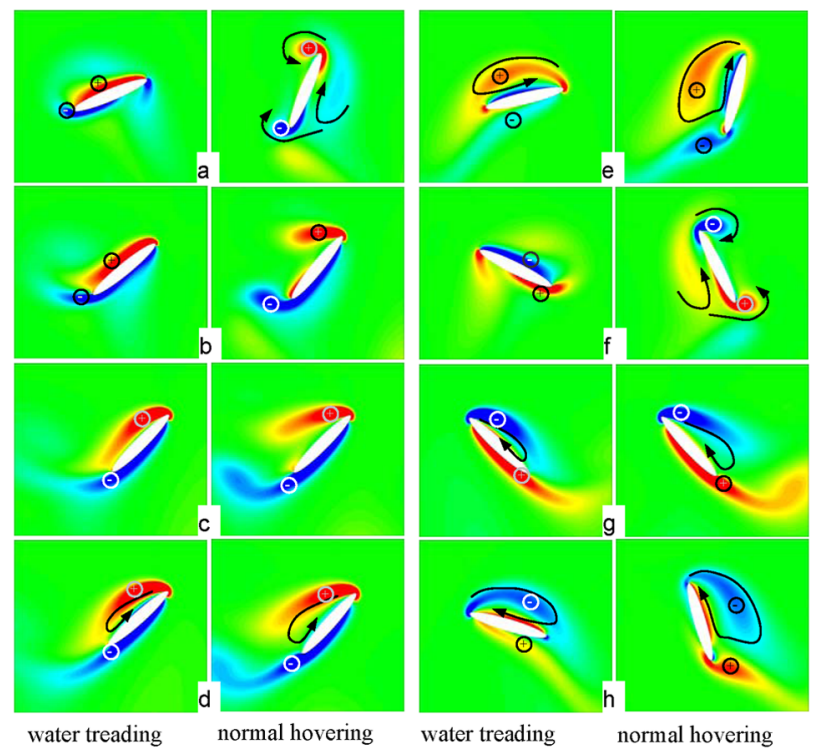

Fig. 9 Vorticity contours for two hovering modes. $h_{a} / c=1.4$, $\alpha_{a}=45 \mathrm{deg}, k=1 / 2.8$, and $R e=100$. (+) = positive vortices; $(-)=$ negative vortices. The flow snapshots a)-h) correspond to the time instants defined in Fig. 8.

reaches the highest value (Fig. 9c). However, as indicated in Fig. 8 , the maximum lift does not appear at the same moment of the maximum pitch angle. This confirms the well-established observation that the flapping aerodynamics cannot be correctly accounted for by the steady-state aerodynamics theory. Beyond midstroke, the airfoil starts to decelerate and pitches down. The flow separates and a large recirculation bubble forms on the upper side of the airfoil (Figs. 9d and 9e) leading to a decrease in lift to the minimum value (Fig. 8 a, at time e). The same pattern is repeated for the backward stroke.

In the normal hovering mode, as in the water trading mode, at the beginning of the forward stroke, the airfoil accelerates and pitches down. The rotation of the airfoil speeds up the flow around the leading and trailing edges, creating a suction zone on the upper side of the airfoil, while the high-pressure stagnation area on the lower side is increased due to the fluid driven from the surroundings by the previously formed vortex (Fig. 9a).

This combination of low- and high-pressure areas leads to an increase in lift at the beginning of the stroke (Fig. 8a, at time a). As the airfoil further rotates downward and accelerates, the fluid is accelerated toward the trailing edge and the high-pressure stagnation area decreases (Fig. 9b) and so does the lift, reaching a local minimum at time $/ T \sim \overline{6} .17$ for the forward stroke and 6.57 for the backward stroke as shown in Fig. 8a. Around the middle of each halfstroke, the airfoil travels at almost constant pitching angle. A recirculation bubble attached to the airfoil forms on the upper surface (Figs. 9c, 9d, and 9g, around time/T 6.3 and 6.8) and helps increase the lift and drag to their maximum values during one complete stroke (Figs. $8 \mathrm{a}$ and $8 \mathrm{~b}$, at times $\mathrm{d}$ and g). After the maximum pitching angle and translation velocity are reached (time $/ T=6.25$ and 6.75) during one half-stroke, the airfoil decelerates and pitches up leading to flow separation on the upper side of the airfoil (Figs. 9e and 9h). The detachment of the large vortical structure from the upper airfoil surface combined with rapid deceleration decreases the circulation and therefore the lift coefficient drops to its minimum value (Fig. $\underline{8}$ a at times e and h).
The force coefficient history for water-treading and normal hovering modes indicates differences in the lift generation mechanism. For both hovering modes, the lift force reaches its maximum value when the airfoil moves near the maximum velocity and maximum pitching angle. Similar maximum lift peak values (Fig. 8a at times $\mathrm{d}$ and g) and flow structures (Figs. 9d and 9g) are observed in this particular time interval (midstroke), suggesting the idea of a similar lift generation mechanism. The vorticity contours (Fig. 9) indicate that the flow is either attached, or with a small recirculation bubble on the upper side of the airfoil and therefore, the delayed-stall mechanism is mainly responsible for generating most of the lift force.

Although the delayed stall is the main lift generation mechanism in the case of the water-treading hovering mode, for the normal hovering mode, the local lift peaks at the beginning of the halfstrokes point out that a wake-capturing mechanism is also a contributing factor, as evidenced by the secondary peak in time history. The presence of the twin-peak characteristics of the lift and drag time histories in the normal hovering mode again confirms that the fluid physics is distinctly time dependent, and cannot be adequately explained by the steady-state theory. Furthermore, for the normal hovering mode, the drag pattern does not mimic that of the lift, as evidenced by the relative magnitudes of the two peaks in lift and drag histories. In contrast, the lift and drag patterns in the watertreading mode show much stronger correspondence, further suggesting the role played by the wake-capturing mechanism in the normal hovering mode. Hence, depending on the detailed kinematics, the lift generation mechanisms at $R e=100$ exhibit different physical mechanisms.

The averaged lift coefficient for both cases is computed as the summation of the lift coefficient over the last three periods divided by the total time. For the water-treading hovering mode an average lift coefficient of 0.77 is obtained, while for the normal hovering mode the average lift coefficient is 0.56 , suggesting that the water-treading mode performs better at $R e=100$ under the given kinematics parameters.

The more significant role of viscosity at low Reynolds numbers reduces the interaction between vortex structures generated during previous strokes, as reflected by the almost symmetric maximum peaks for lift and drag as one can notice in Figs. $\underline{8 a}$ and $\underline{8 b}$.

\section{Effect of Reynolds Number on Aerodynamic Performance in Water-} Treading Mode

Water-Treading Mode

To investigate the Reynolds number effect on the aerodynamic forces and the flow structure, we have computed the hovering aerodynamics of the water-treading mode at $R e=100$ and $\operatorname{Re}=1700$.

Based on the same kinematics of the $R e=100$ case, the aerodynamics of the water-treading mode is assessed. The kinematics and flow parameters for these cases are summarized in Table $\underline{4}$, and the airfoil motion schematic is presented in Fig. $\underline{5 a}$. The lift coefficient history is plotted for two cycles in Fig. 10.

The pressure distributions on the airfoil surface, plotted in Fig. 11, show that near the maximum lift peaks, the high-pressure stagnation area on the lower side of the airfoil is similar in both shape and magnitude for the two Reynolds numbers studied. However, on the upper side of the airfoil, the mild variation of the pressure gradient for the low Reynolds number case (Fig. 11, left-hand panels, times a and c) suggests that the flow is attached, while for the high Reynolds number (Fig. 11, right-hand panels, times a and c) the low-pressure area near the leading edge indicates a recirculation zone

Table 4 Parameters for water-treading hovering mode employed for Reynolds number effect study

\begin{tabular}{lcccrrr}
\hline \hline Hovering mode & $\begin{array}{c}\text { Initial angle } \\
\text { of attack, } \alpha_{0}\end{array}$ & Pitching amplitude, $\alpha_{a}$ & Stroke amplitude, $h_{a} / c$ & Reduced frequency, $k$ & Phase difference, $\varphi$ & Reynolds no., $R e$ \\
\hline 1. Water treading & $0 \mathrm{deg}$ & $45 \mathrm{deg}$ & 1.4 & $1 / 2.8$ & $-\pi / 2$ & 100 \\
2. Water treading & $0 \mathrm{deg}$ & $45 \mathrm{deg}$ & 1.4 & $1 / 2.8$ & $-\pi / 2$ & 1700 \\
\hline \hline
\end{tabular}




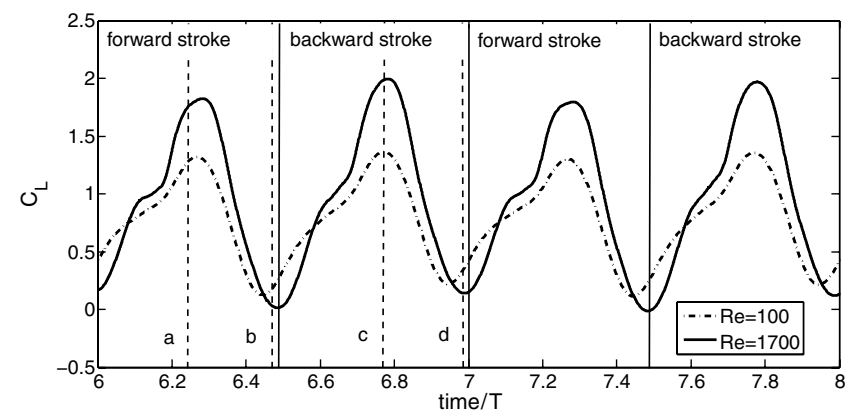

Fig. 10 Lift coefficient for the water-treading mode. $h_{a} / c=1.4$, $\alpha_{a}=45 \mathrm{deg}, k=1 / 2.8$, and $R e=100$ and 1700. The selected normalized time instants are a) 6.25 , b) 6.48 , c) 6.77 , and d) 6.97 .

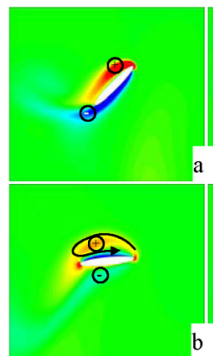

a)

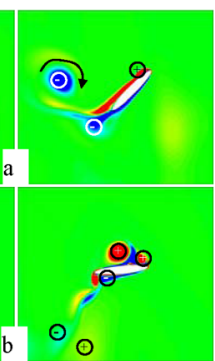

b) c)

d)

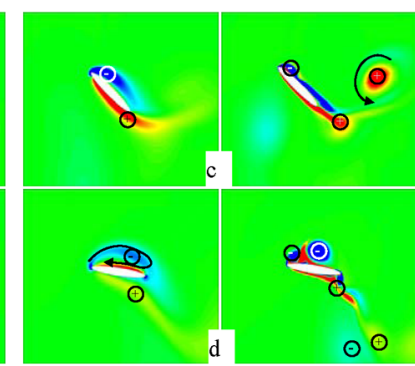

Fig. 12 Vorticity contours for the "water-treading" mode. $h_{a} / c=1.4$, $\alpha_{a}=45 \mathrm{deg}$, and $k=1 / 2.8$. $(+)=$ positive vorticity; $(-)=$ negative vorticity. a), c) $R e=100$; b), d) $R e=1700$. The flow snapshots (a-d) correspond to the time instants defined in Fig. 10.

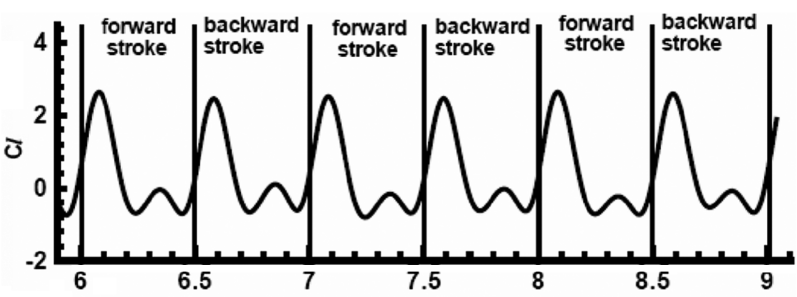

a)

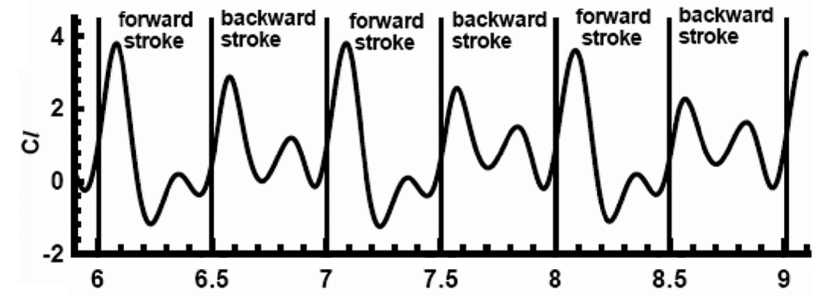

b)

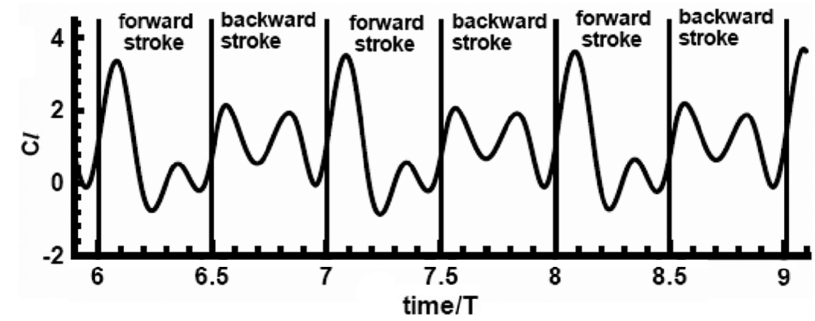

c)

Fig. 13 Lift coefficients at Reynolds number a) $R e=75$, b) $R e=300$, and c) $R e=500$ with $h_{a} / c=0.25, \alpha_{a}=45 \mathrm{deg}$, and $k=2.0$.

In Fig. 13, lift coefficients at three Reynolds numbers are shown. It is clear that force trends of forward and backward strokes are the same at the $R e=75$; at $R e=300$ and 500, the lift coefficient variation is distinctly different between the forward and backward strokes of each cycle. The aerodynamic characteristics regarding the Reynolds number effect in the normal hovering mode are quite different from those in the water-treading mode. In the watertreading mode, while the quantitative differences increase as the Reynolds number increases from 100 to 1700 , no qualitative change was observed. In the normal hovering mode, the qualitatively similar patterns exist at the much lower Reynolds number range, for example, $R e=75$ (see Fig. 7) and 100. The aerodynamic patterns between $R e=75$ and 300 are qualitatively different, suggesting that different physical mechanisms exist.

In Fig. 14, the flowfields of the corresponding positions between the forward and backward strokes in normal flapping mode are plotted. The vortex below the airfoil in Fig. 14a is not found in 
Table 5 Difference of average lift and drag coefficients between forward and backward strokes in the two flapping modes, with flapping amplitude $h_{a} / c=0.25$ at different Reynolds numbers

\begin{tabular}{cccc}
\hline \hline Difference of force coefficient in two strokes of each cycle & $R e=75$ & $R e=300$ & $R e=500$ \\
\hline$\Delta C_{l}$ & 0.002 & 0.325 & 0.33 \\
$\Delta C_{d}$ & 0.045 & 0.105 & 0.125 \\
\hline \hline
\end{tabular}

Fig. $14 \mathrm{~b}$ (corresponding to the backward stroke at the same position and angle of attack). Figure 15 confirms that there is a substantially stronger history effect in the higher Reynolds number regime of the normal hovering mode.

The effect of the Reynolds number on flow structures is complex. For example, Fig. 15 shows that there are two pairs of vortices that shed the airfoil at $\overline{R e}=300$, while there is only one pair of vortex core at $R e=75$. To quantify this asymmetric phenomenon caused by the history effect, the differences of average lift and drag coefficients of the two forward and backward strokes in each cycle, for both normal and water-treading modes, are listed in Table 5 .

The aerodynamic parameters presented in Table 5 suggest that for the normal mode, at $R e=75$, the difference of lift coefficients between forward and backward strokes is very small, while at $R e=300$ and 500, the difference of the lift coefficient is much larger, which indicates a qualitative change appears. This is also proven by the vorticity contours shown Fig. 15 . At $R e=75$, the shedding vortex near the trailing edge is under the airfoil (Fig. 15a) while this vortex is moved to left side in higher Reynolds number cases (Figs. 15b and 15c).

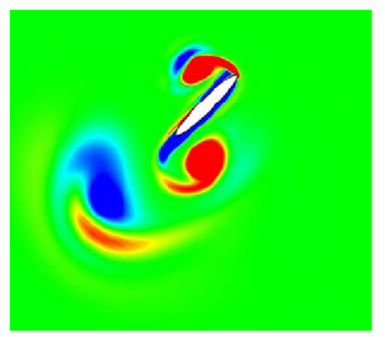

a)

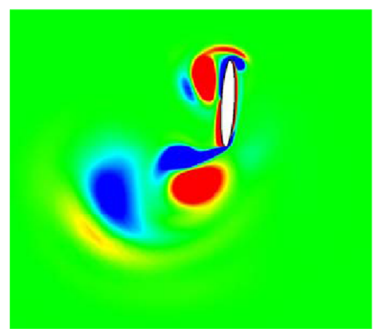

c)

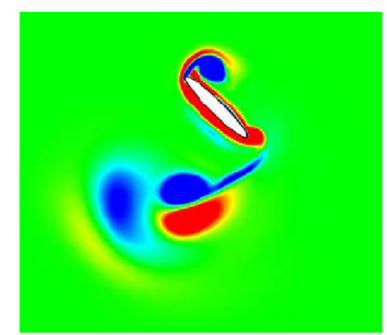

b)

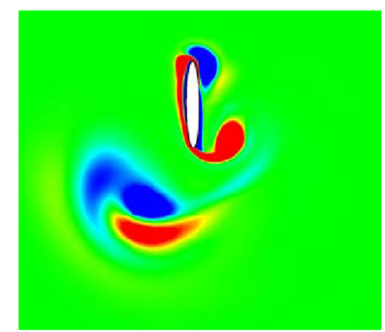

d)

Fig. 14 Vorticity field at corresponding positions of the two sequential strokes a) $($ time $/ T=0.25)$ and $b)($ time $/ T=0.75)$; $)($ time $/ T=0.5)$ and d) $($ time $/ T=1.0)$ with $h_{a} / c=0.25, \alpha_{a}=45 \mathrm{deg}, k=2.0$, and $\boldsymbol{R e}=\mathbf{3 0 0}$.

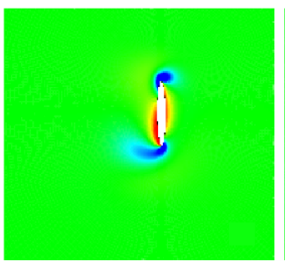

a)

Fig. 15 Vorticity field at the same position at time instant time $/ T=0.5$ under three different Reynolds number with $h_{a} / c=0.25, \alpha_{a}=45 \mathrm{deg}$, and $k=2.0$. a) $R e=100$, b) $R e=300$, and c) $R e=500$.

\section{Summary and Conclusions}

The flow over an elliptic airfoil in hovering motion under different flow parameters was numerically investigated. The unsteady, laminar, incompressible Navier-Stokes equations were solved using a pressure-based Navier-Stokes solver along with a moving grid technique. Two different flapping modes have been investigated at various Reynolds numbers (from 75 to 1700) and reduced frequencies (from 0.25 to 2.0 ).

Within the Reynolds number and reduced frequency ranges investigated, the delayed-stall mechanism is found to be responsible for generating the maximum lift peaks for both hovering modes. On the other hand, the wake-capturing mechanism is identified only in the normal hovering mode, resulting in a twin-peak pattern in both lift and drag. Hence, the kinematics strongly influences the specific physical mechanisms present in lift enhancement.

In addition to the strong role played by the kinematics, the Reynolds number's role has also been clearly identified. At the lower end of the Reynolds number, $\mathcal{O}(100)$, the force patterns and the flow structures in both hovering modes are essentially symmetric during the forward and backward strokes. For a nondimensional flapping amplitude of 1.4 and Reynolds numbers from 100 to 1700, the lift and drag time histories between the forward and backward strokes of the water-treading mode change quantitatively while maintaining similar patterns. On the other hand, in the case of the normal hovering mode, qualitatively different aerodynamic patterns between forward and backward strokes emerge as the Reynolds number increases from 75 to 300 and beyond with a small nondimensional flapping amplitude of 0.25 .

The present study offers insight into the significant roles played by the flapping kinematics, the Reynolds number, and the reduced frequency. Although the current scope is restricted to twodimensional flows and there are additional, important threedimensional aspects that are not addressed, the results have highlighted the interplay between these control parameters as well as the complexity in aerodynamics. It will also be interesting to identify favorable combinations of these flapping parameters from aerodynamics viewpoints to develop suitable strategies for more efficient design of micro air vehicles.

\section{Acknowledgment}

The present work is supported by the Air Force Office of Scientific Research (AFOSR).

\section{References}

[1] Shyy, W., Berg, M., and Ljungqvist, D., "Flapping and Flexible Wings for Biological and Micro Air Vehicles," Progress in Aerospace Sciences, Vol. 35, No. 5, 1999, pp. 455-506. doi:10.1016/S0376-0421(98)00016-5

[2] Lehmann, F.-O., "The Mechanisms of Lift Enhancement in Insect Flight," Naturwissenschaften, Vol. 91, No. 3, 2004, pp. 101-122. doi:10.1007/s00114-004-0502-3

[3] Norberg, U. M., Vertebrate Flight: Mechanics, Physiology, Morphology, Ecology and Evolution, Springer-Verlag, Berlin, 1990.

[4] Jones, K. D., and Platzer, F. M., "Bio-Inspired Design of FlappingWing Micro Air Vehicles-An Engineer's Perspective," AIAA Paper 2006-0037, 2006.

[5] Viieru, D., Tang, J., Lian, Y., Liu, H., and Shyy, W., "Flapping and Flexible Wing Aerodynamics of Low Reynolds Number Flight Vehicles," AIAA Paper 2006-0503, 2006.

[6] Wang, Z. J., "Dissecting Insect Flight," Annual Review of Fluid Mechanics, Vol. 37, Jan. 2005, pp. 183-210. doi:10.1146/annurev.fluid.36.050802.121940 
[7] Mueller, T. J. (ed.), Fixed and Flapping Wing Aerodynamics for Micro Air Vehicle Applications, Progress in Astronautics and Aeronautics Series, AIAA, Reston, VA, 2001, Vol. 195.

[8] Azuma, A., The Biokinetics of Flying and Swimming, Springer-Verlag, Tokyo, 1992.

[9] Pennycuick, C. J., Bird Flight Performance: A Practical Calculation Manual, Oxford Univ. Press, Oxford, U.K. 1989.

[10] Tennekes, H., The Simple Science of Flight (from Insects to Jumbo Jets), MIT Press, Boston, MA, 1996.

[11] Ellington, C. P., "The Aerodynamics of Hovering Insect Flight. 1. The Quasi-Steady Analysis," Philosophical Transactions of the Royal Society of London, Series B: Biological Sciences, Vol. 305, No. 1122, 1984, pp. 1-15. doi:10.1098/rstb.1984.0049

[12] Weis-Fogh, T., "Quick Estimates of Flight Fitness in Hovering Animals, Including Novel Mechanisms for Lift Production," Journal of Experimental Biology, Vol. 59, No. 1, 1973, pp. 169-230.

[13] Ellington, C. P., Van den Berg, C., Willmott, A. P., and Thomas, A. L. R., "Leading-Edge Vortices in Insect Flight," Nature (London), Vol. 384, No. 6610, 1996, pp. 626-630. doi: $10.1038 / 384626 \mathrm{a} 0$

[14] Freymuth, P., "Propulsive Vortical Signatures of Plunging and Pitching Airfoils," AIAA Paper 88-323, 1988.

[15] Dickinson, M. H., Lehmann, F.-O., and Sane, S. P., "Wing Rotation and the Aerodynamic Basis of Insect Flight," Science, Vol. 284, No. 5422, 1999, pp. 1954-1960. doi:10.1126/science.284.5422.1954

[16] Liu, H., and Kawachi, K., "A Numerical Study of Insect Flight," Journal of Computational Physics, Vol. 146, No. 1, 1998, pp. 124-156. doi:10.1006/jcph.1998.6019

[17] Van den Berg, C., and Ellington, C. P., "The Three-Dimensional Leading-Edge Vortex of a 'Hovering' Model Hawkmoth," Philosophical Transactions of the Royal Society of London, Series B: Biological Sciences, Vol. 352, No. 1351, 1997, pp. 329-340. doi:10.1098/rstb.1997.0024

[18] Sun, M., and Tang, J., "Unsteady Aerodynamic Force Generation by a Model Fruit Fly Wing in Flapping Motion," Journal of Experimental Biology, Vol. 205, No. 1, 2002, pp. 55-70.

[19] Ramamurti, R., and Sandberg, W. C., "A Three-Dimensional Computational Study of the Aerodynamic Mechanisms of Insect Flight," Journal of Experimental Biology, Vol. 205, No. 10, 2002, pp. 1507-1518.

[20] Wang, Z. J., Birch, J. M., and Dickinson, M. H., "Unsteady Forces and Flows in Low Reynolds Number Hovering Flight: Two-Dimensional Computations vs Robotic Wing Experiments," Journal of Experimental Biology, Vol. 207, No. 3, 2004, pp. 449-460. doi:10.1242/jeb.00739

[21] Kurtulus, D. F., Farcy, A., and Alemdaroglu, N., "Unsteady Aerodynamics of Flapping Airfoil in Hovering Flight at Low Reynolds Numbers," AIAA Paper 2005-1356, 2005.

[22] Freymuth, P., "Thrust Generation by an Airfoil in Hover Modes," Experiments in Fluids, Vol. 9, Nos. 1-2, Jan. 1990, pp. 17-24. doi:10.1007/BF00575331

[23] Tannehill, J. C., Anderson, D. A., and Pletcher, R. H., Computational Fluid Mechanics and Heat Transfer, 2nd ed., Taylor and Francis, Bristol, U.K., 1997.

[24] Thakur, S., Wright, J., and Shyy, W., "Stream: A Computational Fluid Dynamics and Heat Transfer Navier-Stokes Solver. Theory and
Applications," Streamline Numerics, Inc. and Computational ThermoFluids Laboratory, Department of Mechanical and Aerospace Engineering, Gainesville, FL, 2002.

[25] Shyy, W., Computational Modeling for Fluid Flow and Interfacial Transport, edited by A. S. Mujumdar, Vol. 5, Series in Transport Processes in Engineering, Elsevier, Amsterdam, The Netherlands, 1994.

[26] Patankar, S. V., and Spalding, D. B., "A Calculation Procedure for Heat, Mass and Momentum Transfer in Three-Dimensional Parabolic Flows," International Journal of Heat and Mass Transfer, Vol. 15, No. 10 , Oct. 1972 , pp. 1787-1806. doi:10.1016/0017-9310(72)90054-3

[27] Van Doormaal, J. P., and Raithby, G. D., "Enhancements of the Simple Method for Predicting Incompressible Fluid Flows," Numerical Heat Transfer, Vol. 7, April-June 1984, pp. 147-163. doi:10.1080/01495728408961817

[28] Shyy, W., Udaykumar, H. S., Madhukar, M. R., and Richard, W. S., Computational Fluid Dynamics with Moving Boundarie, Series in Computational and Physical Processes in Mechanics and Thermal Sciences, Taylor and Francis, Washington, D.C., 1996.

[29] Thomas, P. D., and Lombard, K., "Geometric Conservation Law and Its Applications to Flow Computations on Moving Grids," AIAA Journal, Vol. 17, No. 10, 1979, pp. 1030-1037.

[30] Schuster, D., Vadyak, J., and Atta, E., "Static Aeroelastic Analysis of Fighter Aircraft Using a Three-Dimensional Navier-Stokes Algorithm," Journal of Aircraft, Vol. 27, No. 9, 1990, pp. 820-825.

[31] Batina, J. T., "Unsteady Euler Airfoil Solutions Using Unstructured Dynamic Meshes," AIAA Paper 1989-115, 1989.

[32] Robinson, B. A., Yang, H. T. Y., and Batina, J. T., "Aeroelastic Analysis of Wings Using Euler Equations with a Deforming Mesh," Journal of Aircraft, Vol. 28, No. 11, 1991, pp. 781-788.

[33] Eriksson, L. E., "Generation of Boundary-Conforming Grids Around Wing-Body Configurations Using Transfinite Interpolation," AIAA Journal, Vol. 20, No. 10, 1982, pp. 1313-1320.

[34] Hartwich, P. M., and Agrawal, S., "Method for Perturbing Multiblock Patched Grids in Aeroelastic and Design Optimization Applications," AIAA Paper 1997-2038, 1997.

[35] Lian, Y., Steen, J., Trygg-Wilander, M., and Shyy, W., "Low Reynolds Number Turbulent Flows Around a Dynamically Shaped Airfoil," Computers and Fluids, Vol. 32, No. 3, 2003, pp. 287-303. doi:10.1016/S0045-7930(01)00087-1

[36] Schlichting, H., Boundary Layer Theory, 7th ed., McGraw-Hill, New York, 1979.

[37] Stewartson, K., "Multistructured Boundary Layers on Flat Plates and Related Bodies," Advances in Applied Mathematics, Vol. 14, 1974, pp. 146-239.

[38] Messiter, A. F., "Boundary Layer Flow Near the Trailing Edge of a Flat Plate," SIAM Journal of Applied Mathematics, Vol. 18, No. 1, Jan. 1970 , pp. 241-257.

[39] Stokes, G. G., "On the Effect of the Internal Friction of Fluids on the Motion of Pendulums," Cambridge Philosophical Transactions, Vol. 9, 1851, pp. 8-106.

[40] White, F. M., Viscous Fluid Flow, 2nd ed., McGraw-Hill, New York, 1991. 